\title{
œcreative
}

\section{3-pyramidal Steiner triple systems ${ }^{*}$}

\author{
Marco Buratti \\ Dipartimento di Matematica e Informatica, \\ Università di Perugia, via Vanvitelli 1, 06123 Perugia, Italy \\ Gloria Rinaldi \\ Dipartimento di Scienze e Metodi dell'Ingegneria, \\ Università di Modena e Reggio Emilia, via Amendola 2, 42122 Reggio Emilia, Italy \\ Tommaso Traetta \\ Department of Mathematics, Ryerson University, Toronto (ON) M5B 2K3, Canada \\ Received 21 December 2015, accepted 30 March 2016, published online 19 February 2017
}

\begin{abstract}
A design is said to be $f$-pyramidal when it has an automorphism group which fixes $f$ points and acts sharply transitively on all the others. The problem of establishing the set of values of $v$ for which there exists an $f$-pyramidal Steiner triple system of order $v$ has been deeply investigated in the case $f=1$ but it remains open for a special class of values of $v$. The same problem for the next possible $f$, which is $f=3$, is here completely solved: there exists a 3-pyramidal Steiner triple system of order $v$ if and only if $v \equiv 7,9,15(\bmod 24)$ or $v \equiv 3,19(\bmod 48)$.
\end{abstract}

Keywords: Steiner triple system, group action, difference family, Skolem sequence, Langford sequence.

Math. Subj. Class.: 51E10, 20B25, 05B07, 05B10

\section{Introduction}

A Steiner triple system of order $v$, briefly $\operatorname{STS}(v)$, is a pair $(V, \mathcal{B})$ where $V$ is a set of $v$ points and $\mathcal{B}$ is a set of 3-subsets (blocks or triples) of $V$ with the property that any two distinct points are contained in exactly one block. Apart from the trivial case $v=0$ in

\footnotetext{
* Research performed within the activity of INdAM-GNSAGA with the financial support of the italian Ministry MIUR, project "Combinatorial Designs, Graphs and their Applications".

E-mail addresses: buratti@dmi.unipg.it (Marco Buratti), gloria.rinaldi@unimore.it (Gloria Rinaldi), tommaso.traetta@ryerson.ca, traetta.tommaso@gmail.com (Tommaso Traetta)
} 
which both $V$ and $\mathcal{B}$ are empty, it is well known that a $\operatorname{STS}(v)$ exists if and only if $v \equiv 1$ or $3(\bmod 6)$. For general background on STSs we refer to [7].

Steiner triple systems having an automorphism with a prescribed property or an automorphism group with a prescribed action have drawn much attention since a long time. It was proved by Peltesohn [15] that a $\operatorname{STS}(v)$ with an automorphism cyclically permuting all points, briefly a cyclic $\operatorname{STS}(v)$, exists for any possible $v$ but $v \neq 9$. The existence question for a $\operatorname{STS}(v)$ with an involutory automorphism fixing exactly one point, briefly a reverse $\operatorname{STS}(v)$, has been settled by means of three different contributions of Doyen [9], Rosa [18] and Teirlinck [21]; it exists if and only if $v \equiv 1,3,9,19(\bmod 24)$. In [16] Phelps and Rosa proved that there exists a $\operatorname{STS}(v)$ with an automorphism cyclically permuting all but one point, briefly a 1-rotational $\operatorname{STS}(v)$, if and only if $v \equiv 3$ or $9(\bmod 24)$.

Note that a cyclic or 1-rotational STS may be viewed as a STS with a cyclic automorphism group acting sharply transitively on all points or all but one point, respectively. Thus one may ask, more generally, for a STS with an automorphism group $G$ having the same kind of action without the request that $G$ be cyclic.

Speaking of a regular $\operatorname{STS}(v)$ we mean a $\operatorname{STS}(v)$ for which there is at least one group $G$ acting sharply transitively on the points. Also, speaking of a 1-rotational $\operatorname{STS}(v)$ we mean a $\operatorname{STS}(v)$ with at least one automorphism group acting regularly on all but one points.

The only STS(9), which is the point-line design associated with the affine plane over $\mathbb{Z}_{3}$, is clearly regular under $\mathbb{Z}_{3}^{2}$. Thus, in view of Peltesohn's result, there exists a regular $\operatorname{STS}(v)$ for any admissible $v$.

The problem of determining the set of values of $v$ for which there exists a 1-rotational $\operatorname{STS}(v)$ (under some group) has been deeply investigated in [2, 4]. Such a STS is necessarily reverse so that $v$ must be congruent to $1,3,9$, or $19(\bmod 24)$. For the cases $v \equiv 3$ or $9(\bmod 24)$ the existence clearly follows from the result by Phelps and Rosa. In the case $v \equiv 19(\bmod 24)$ we do not have existence only when $v=6 P Q+1$ with $P=1$ or a product of pairwise distinct primes congruent to $5(\bmod 12)$ and with $Q$ a product of an odd number of pairwise distinct primes congruent to $11(\bmod 12)$. The most difficult case is $v \equiv 1(\bmod 24)$ where the existence remains still uncertain only when all the following conditions are simultaneously satisfied: $v=\left(p^{3}-p\right) n+1 \equiv 1(\bmod 96)$ with $p$ a prime; $n \not \equiv 0(\bmod 4)$; the odd part of $v-1$ is square-free and without prime factors $\equiv 1(\bmod 6)$.

Of course one might consider the more specific problems of determining all groups $G$ for which there exists a STS which is regular under $G$ and all groups $G$ for which there exists a STS which is 1-rotational under $G$. These problems appear at this moment quite hard. The same problems can be relaxed by asking for which $v$ there is a $\operatorname{STS}(v)$ which is regular (1-rotational) under a group belonging to an assigned general class. For instance, the first author proved that there exists a $\operatorname{STS}(v)$ which is 1-rotational under some abelian group if and only if either $v \equiv 3,9(\bmod 24)$ or $v \equiv 1,19(\bmod 72)$. Also, Mishima [14] proved that there exists a $\operatorname{STS}(v)$ which is 1-rotational under a dicyclic group if and only if $v \equiv 9(\bmod 24)$.

We have quoted only the results which are closer to the problem that we are going to study in this paper. Indeed the literature on Steiner triple systems and their automorphism groups is quite wide. For example, results concerning the full automorphism group of a STS have been obtained by Mendelsohn [13] and Lovegrove [12].

Now we want to consider the problem of determining the set of values of $v$ for which there exists a $\operatorname{STS}(v)$ with an automorphism group fixing $f$ points and acting sharply transitively on the other $v-f$ points. Such a STS will be called $f$-pyramidal. Of course the 
cases $f=0$ and $f=1$ correspond, respectively, to the regular and 1-rotational STSs discussed above. It is natural to study the next possible case that is $f=3$. This is because, as we are going to see in the next lemma, the fixed points of an $f$-pyramidal $\operatorname{STS}(v)$ form a subsystem of order $f$ so that, for $f \neq 0$, we have $f \equiv 1$ or $3(\bmod 6)$; also, if $f \neq v$, then $f<v / 2$.

Lemma 1.1. A necessary condition for the existence of an $f$-pyramidal $\operatorname{STS}(v)$ is that $f=0$ or $f \equiv 1,3(\bmod 6)$, and $f=v$ or $f<v / 2$.

Proof. Let $(V, \mathcal{B})$ be an $f$-pyramidal $\operatorname{STS}(v)$ under the action of a group $G$. Assume, w.l.o.g., that $G$ is additive and let $F=\left\{\infty_{1}, \ldots, \infty_{f}\right\}$ be the set of points fixed by $G$. Obviously $G$ has order $v-f$, the set of points $V$ can be identified with $F \cup G$, and the action of $G$ on $V$ can be identified with the addition on the right with the assumption that $\infty_{i}+g=\infty_{i}$ for each $\infty_{i} \in F$ and each $g \in G$. If a block $B \in \mathcal{B}$ contains two distinct fixed points, say $\infty_{i}$ and $\infty_{j}$, then $B+g=B$ for every $g \in G$ otherwise $\mathcal{B}$ would have two distinct blocks, $B$ and $B+g$, passing through the two points $\infty_{i}$ and $\infty_{j}$. So, the third vertex of $B$ is also fixed by $G$. It easily follows that all blocks of $\mathcal{B}$ contained in $F$ form a $\operatorname{STS}(f)$ so that we have $f=0$ or $f \equiv 1,3(\bmod 6)$, and $f=v$ or $f<v / 2$.

The main result of this paper is a complete solution to the existence problem for a 3-pyramidal $\operatorname{STS}(v)$.

Theorem 1.2. There exists a 3-pyramidal $\operatorname{STS}(v)$ if and only if $v \equiv 7,9,15(\bmod 24)$ or $v \equiv 3,19(\bmod 48)$.

The "if part" of this theorem will be proved in Section 3 which therefore will give non-existence results: for $v \equiv 1,13,21(\bmod 24)$ or $v \equiv 27,43(\bmod 48)$ there is no 3-pyramidal $\operatorname{STS}(v)$. The "only if part" will be proved in Section 4 where we will give an explicit construction of a 3 -pyramidal $\operatorname{STS}(v)$ whenever $v \equiv 7,9,15(\bmod 24)$ or $v \equiv 3,19$ $(\bmod 48)$.

First, in the next section, we have to translate our problem into algebraic terms: any $f$-pyramidal STS is completely equivalent to a suitable difference family.

\section{Difference families and pyramidal STSs}

As a natural generalization of the concept of a relative difference set [17], the first author introduced [3] difference families in a group $G$ relative to a subgroup of $G$ or, even more generally [5], relative to a partial spread of $G$. By a partial spread of a group $G$ one means a set $\Sigma$ of subgroups of $G$ whose mutual intersections are all trivial. One omits the attribute "partial" in the special case that the subgroups of $\Sigma$ cover all $G$. Let $\Sigma$ be a partial spread of an additively written group $G$, let $\mathcal{F}$ be a set of $k$-subsets $G$, and let $\Delta \mathcal{F}$ be the list of all possible differences $x-y$ with $(x, y)$ an ordered pair of distinct elements of a member of $\mathcal{F}$. One says that $\mathcal{F}$ is a $(G, \Sigma, k, 1)$-difference family (DF) if every group element appears 0 or 1 times in $\Delta \mathcal{F}$ according to whether it belongs or does not belong to some member of $\Sigma$, respectively. We say that $\Sigma$ is of type $\left\{d_{1}^{e_{1}}, \ldots, d_{n}^{e_{n}}\right\}$ if this is the multiset (written in "exponential" notation) of the orders of all subgroups belonging to $\Sigma$ and we speak of a $\left(G,\left\{d_{1}^{e_{1}}, \ldots, d_{n}^{e_{n}}\right\}, k, 1\right)-\mathrm{DF}$.

It is obvious that any $\operatorname{STS}(v)$ is $v$-pyramidal under the trivial group. The following theorem explains how to construct an $f$-pyramidal $\operatorname{STS}(v)$ with $f<v / 2$. It generalizes Theorem 1.1 in [3] which corresponds to the case $f=1$. 
Theorem 2.1. There exists an $f$-pyramidal $\operatorname{STS}(v)$ with $f<v / 2$ if and only if there exists $a\left(G,\left\{2^{f}, 3^{e}\right\}, 3,1\right)$-DF for a suitable group $G$ of order $v-f$ with exactly $f$ involutions, and a suitable integer $e$.

Proof. $(\Longrightarrow)$. Let $(V, \mathcal{B})$ be an $f$-pyramidal $\operatorname{STS}(v)$ under an additive group $G$. We can assume that $V=F \cup G$ with $F$ and the action of $G$ on $V$ defined as in Lemma 1.1. For $1 \leq i \leq f$, let $B_{i}=\left\{\infty_{i}, 0, x_{i}\right\}$ be the block of $\mathcal{B}$ containing the points $\infty_{i}$ and 0 . We have $B_{i}-x_{i}=\left\{\infty_{i},-x_{i}, 0\right\}$ so that both $B_{i}$ and $B_{i}-x_{i}$ contain the points $\infty_{i}$ and 0 . It necessarily follows that $B_{i}-x_{i}=B_{i}$, hence $-x_{i}=x_{i}$ which means that $x_{i}$ is an involution. Conversely, if $x$ is an involution of $G$ and $B=\{0, x, y\}$ is the block through 0 and $x$, then $B+x=\{x, 0, y+x\}$ would also contain 0 and $x$ so that $B+x=B$. This means that $y+x=y$ and this is possible only if $y \in F$. Hence $y=\infty_{i}$ and $x=x_{i}$ for a suitable $i$. We conclude that $\left\{x_{1}, \ldots, x_{f}\right\}$ is the set of all involutions of $G$.

Let $\mathcal{F}$ be a complete system of representatives for the $G$-orbits on the blocks of $\mathcal{B}$ with trivial $G$-stabilizer. Reasoning as in the "if part" of Theorem 2.2 in [4], one can see that $\mathcal{F}$ is a $(G, \Sigma, 3,1)$-DF where $\Sigma$ is the partial spread of $G$ consisting of all 2-subgroups $\left\{0, x_{i}\right\}$ ( $i=1, \ldots, f$ ) of $G$ and all 3-subgroups of $G$ belonging to $\mathcal{B}$.

$(\Longleftarrow)$. Now assume that $f \equiv 1$ or $3(\bmod 6)$ and that $\mathcal{F}$ is a $(G, \Sigma, 3,1)$-DF with $G$ a group of order $v-f$ having exactly $f$ involutions and with $\Sigma$ a partial spread of $G$ of type $\left\{2^{f}, 3^{e}\right\}$.

Take an $f$-set $F=\left\{\infty_{1}, \ldots, \infty_{f}\right\}$ disjoint with $G$ and let $\left(F, \mathcal{B}_{\infty}\right)$ be any $\operatorname{STS}(f)$ (which exists because we assumed that $f \equiv 1$ or $3(\bmod 6)$ ). For $i=2,3$, let $\Sigma_{i}$ be the set of subgroups of order $i$ belonging to $\Sigma$. Set $\Sigma_{2}=\left\{S_{i} \mid 1 \leq i \leq f\right\}$ and $\Sigma_{2}^{+}=$ $\left\{S_{i} \cup\left\{\infty_{i}\right\} \mid 1 \leq i \leq f\right\}$. Then, as in the "only if part" of Theorem 2.2 in [4], one can see that

$$
\Sigma_{2}^{+} \cup \Sigma_{3} \cup \mathcal{F} \cup \mathcal{B}_{\infty}
$$

is a complete system of representatives for the block-orbits of a 3-pyramidal STS $(v)$ under the action of $G$ on $F \cup G$ defined as in the proof of Lemma 1.1.

Remark 2.2. Considering that "cyclic STS" means "0-pyramidal STS under the cyclic group", as a very special case of the above theorem we have the well known fact that any cyclic $\operatorname{STS}(6 n+1)$ is equivalent to a $\left(\mathbb{Z}_{6 n+1},\{1\}, 3,1\right)$-DF and that any cyclic $\operatorname{STS}(6 n+3)$ is equivalent to a $\left(\mathbb{Z}_{6 n+3},\{3\}, 3,1\right)$-DF.

Example 2.3. The empty-set clearly is a $\left(\mathbb{Z}_{2}^{n},\left\{2^{2^{n}-1}\right\}, 3,1\right)$-DF since every non-zero element of $\mathbb{Z}_{2}^{n}$ is an involution. It is not difficult to see that one of the associated $\left(2^{n}-1\right)$ pyramidal STS $\left(2^{n+1}-1\right)$ is the point-line design of the $n$-dimensional projective geometry over $\mathbb{Z}_{2}$.

Let $\mathbb{D}_{2 n}$ be the dihedral group of order $2 n$, namely the group with defining relations $\mathbb{D}_{2 n}=\left\langle x, y \mid y^{2}=x^{n}=1 ; y x=x^{-1} y\right\rangle$. We give here an example of a STS $(3 f)$ which is $f$-pyramidal under $\mathbb{D}_{2 f}$.

Example 2.4. Let $f \equiv 1$ or $3(\bmod 6)$ but $f \neq 9$. Let $\phi: \mathbb{Z}_{f} \longrightarrow \mathbb{D}_{2 f}$ be the group monomorphism defined by $\phi(i)=x^{i}$ for each $i \in \mathbb{Z}_{f}$. The hypotesis on $f$ guarantees, in view of Peltesohn's result, that there exists a cyclic STS $(f)$. Thus, by Remark 2.2, there exists a $\left(\mathbb{Z}_{f},\{1\}, 3,1\right)$-DF or a $\left(\mathbb{Z}_{f},\{3\}, 3,1\right)$-DF $\mathcal{F}$ according to whether $f \equiv 1$ or $3(\bmod$ $6)$, respectively. It is then obvious that $\{\phi(B) \mid B \in \mathcal{F}\}$ is a $\left(\mathbb{D}_{2 f},\left\{2^{f}, 3^{e}\right\}, 3,1\right)$-DF with $e=0$ or 1 , respectively. 
Thus there exists an $f$-pyramidal $\operatorname{STS}(3 f)$ under the dihedral group $\mathbb{D}_{2 f}$ for any $f \equiv 1$ or $3(\bmod 6)$ but $f \neq 9$.

If, in the above example, we put $f=3$, we obtain a representation of the affine plane of order 3 as a 3 -pyramidal STS $(9)$ under $\mathbb{D}_{6}$. In this case the difference family $\mathcal{F}$ is empty because it is relative to a spread which is not partial; its subgroups $\{1, y\},\{1, x y\},\left\{1, x^{2} y\right\}$ and $\left\{1, x, x^{2}\right\}$ cover indeed all elements of $\mathbb{D}_{6}$. Following the instructions of the "only if part" of Theorem 2.1 the blocks of the STS(9) are:

$$
\begin{array}{ccc}
\left\{\infty_{1}, \infty_{2}, \infty_{3}\right\}, & \left\{1, x, x^{2}\right\}, & \left\{y, x y, x^{2} y\right\}, \\
\left\{\infty_{1}, 1, y\right\}, & \left\{\infty_{1}, x, x^{2} y\right\}, & \left\{\infty_{1}, x^{2}, x y\right\}, \\
\left\{\infty_{2}, 1, x y\right\}, & \left\{\infty_{2}, x, y\right\}, & \left\{\infty_{2}, x^{2}, x^{2} y\right\}, \\
\left\{\infty_{3}, 1, x^{2} y\right\}, & \left\{\infty_{3}, x, x y\right\}, & \left\{\infty_{3}, x^{2}, y\right\} .
\end{array}
$$

In Section 4 we will make use of $\mathbb{D}_{6}$ again, for the construction of a 3-pyramidal $\operatorname{STS}(24 n+9)$ under $\mathbb{D}_{6} \times \mathbb{Z}_{4 n+1}$.

\section{The "if part"}

In this section we determine the values of $v$ for which a 3-pyramidal $\operatorname{STS}(v)$ cannot exist, we namely prove the "if part" of the main result Theorem 1.2. For this, we need two lemmas about elementary group theory.

Lemma 3.1. If $G$ is a group of order $24 n+18$, then $G$ has a subgroup of index 2 .

Proof. It is well known that a group of order twice an odd number has a subgroup of index 2. See, for example, [19, Exercise 262].

The next lemma makes use of the so-called "Burnside normal $p$-complement theorem" which is here recalled (see [19, Theorem 6.17]).

Theorem 3.2. Let $P$ be a Sylow p-subgroup of a finite group $G$. If $C_{G}(P)=N_{G}(P)$, then $P$ has a normal complement in $G$.

Lemma 3.3. If $G$ is a group of order $16 n+8$ containing exactly 3 involutions, then $G$ has a subgroup of index 2 containing exactly one involution.

Proof. Let $j_{1}, j_{2}$, and $j_{3}$ be the three involutions of $G$ and let $H=\left\langle j_{1}, j_{2}, j_{3}\right\rangle$ be the group they generate. We point out that $H$ is a normal subgroup of $G$ since it is generated by all the elements of order 2 . Now, let $P$ be a 2 -Sylow subgroup of $G$. As $P$ has order 8 and $G$ contains exactly three involutions, by taking into account the classification of the groups of order 8 , we have three possibilities: namely $P$ is either isomorphic to the group $\mathbb{Z}_{4} \times \mathbb{Z}_{2}$ or $P$ contains exactly one involution, i.e., it is $P \simeq \mathbb{Z}_{8}$ or $P \simeq Q_{8}$ (the quaternion group of order 8).

First of all we prove that it is necessarily $P \simeq \mathbb{Z}_{4} \times \mathbb{Z}_{2}$. By contradiction, assume that $P$ contains exactly one involution. It is known that, in general, any two involutions of $G$ generate a dihedral group; also, a dihedral group of order $2 h$ contains at least $h$ involutions. Therefore, either $\left\langle j_{1}, j_{2}\right\rangle \simeq \mathbb{D}_{4} \simeq \mathbb{Z}_{2} \times \mathbb{Z}_{2}$ or $\left\langle j_{1}, j_{2}\right\rangle \simeq \mathbb{D}_{6}$. In both cases, $\left\langle j_{1}, j_{2}\right\rangle$ has three involutions, hence $j_{3} \in\left\langle j_{1}, j_{2}\right\rangle$ and $H=\left\langle j_{1}, j_{2}\right\rangle$. Since $P$ contains just one involution, it is necessarily $H=\mathbb{D}_{6}$, otherwise $P$ should contain a subgroup isomorphic to $\mathbb{Z}_{2} \times \mathbb{Z}_{2}$. 
Let $T$ be the subgroup of $H$ of order 3 . As usual, we denote by $N_{G}(T)$ and $C_{G}(T)$ the normalizer and the centralizer of $T$ in $G$, respectively. By the $N / C$-theorem, the quotient group $N_{G}(T) / C_{G}(T)$ is isomorphic to a subgroup of $A u t(T) \simeq \mathbb{Z}_{2}$. Since $T$ is the unique subgroup of $H$ of order $3, T$ is characteristic in $H$ and then it is normal in $G$, that is $N_{G}(T)=G$. Therefore, either $C_{G}(T)=G$ or $C_{G}(T)$ is a subgroup of $G$ of index 2 . In both cases, $C_{G}(T)$ is a normal subgroup of $G$ of even order. The three involutions of $G$ are pairwise conjugate, hence, they are contained in any normal subgroup of $G$ of even order and then in $C_{G}(T)$. It then follows that $T$ is central in $H$ contradicting the fact that $H$ is dihedral.

We conclude that $P \simeq \mathbb{Z}_{4} \times \mathbb{Z}_{2}$; in particular, $P$ is abelian and contains a subgroup $Q \simeq \mathbb{Z}_{4}$; also, $P \supseteq H \simeq \mathbb{Z}_{2} \times \mathbb{Z}_{2}$. To prove the assertion, we need to show that $G$ has a normal subgroup $O$ of order $2 n+1$. In fact, the semidirect product of $O$ and $Q$ will be a subgroup of $G$ of index 2 containing exactly one involution.

Recall that $\left|G: C_{G}\left(j_{i}\right)\right|$ is the size of $c l\left(j_{i}\right)$, the conjugacy class of $j_{i}$ in $G$, which cannot exceed the total number of involutions in $G$ hence, $\left|G: C_{G}\left(j_{i}\right)\right| \leq 3$ for any $i=1,2,3$. On the other hand, $P$ is abelian hence, $P \leq C_{G}\left(j_{i}\right)$ for any $i=1,2,3$. It then follows that either $\left|G: C_{G}\left(j_{i}\right)\right|=1$ for any $i=1,2,3$ or $\left|G: C_{G}\left(j_{i}\right)\right|=3$ for any $i=1,2,3$.

We first deal with the former case in which all involutions of $G$ are central. Since $G / H$ has order $2 d, d$ odd, then it has a subgroup of index 2 (see for example [19, Exercise 262]). In other words, there exists a normal subgroup $N$ of $G$ of index 2 which contains $H$. Since $H$ is a central 2-Sylow subgroup of $N$, by Theorem 3.2, $H$ has a normal complement $O$ in $N$; in particular, $O$ has order $2 n+1$. Now, suppose the existence of another subgroup $K$ of $N$ of order $2 n+1$. Then $K \cap O$ is normal in $K$ and the quotient $Q_{1}=K /(K \cap O)$ should be isomorphic to $Q_{2}=(K+O) / O$. However, $Q_{1}$ has odd order, while $Q_{2}$ has even order. Therefore, $O$ is the only subgroup of $N$ of order $2 n+1$, hence it is normal in $G$.

We finally consider the case $\left|G: C_{G}\left(j_{i}\right)\right|=3$ for any $i=1,2,3$. By the N/C-theorem, $G / C_{G}(H)$ is isomorphic to a subgroup of $\operatorname{Aut}(H) \simeq \mathbb{D}_{6}$. Since $P \leq C_{G}(H) \leq C_{G}\left(j_{i}\right)$, we have that $C_{G}(H)=C_{G}\left(j_{i}\right)$ for any $i=1,2,3$. Now note that $C_{G}(H)$ satisfies the same assumption as $G$ and all involutions are central in $C_{G}(H)$. Therefore, we can proceed as in the previous case to show that there is a normal subgroup $\Omega$ of $C_{G}(H)$ of order $\frac{2 n+1}{3}$. As before, it is not difficult to check that $\Omega$ is the only subgroup of $C_{G}(H)$ of order $\frac{2 n^{3}+1}{3}$, therefore it is normal in $G$.

Set now $\bar{G}=G / \Omega$ and $\bar{P}=C_{G}(H) / \Omega$. Note that $\bar{P} \simeq P, \bar{P}$ is normal in $\bar{G}$ (i.e., $\left.N_{\bar{G}}(\bar{P})=\bar{G}\right)$ and $\bar{G} / \bar{P}$ has order 3 . Also, since $\bar{P}$ is abelian, then $\bar{P} \leq C_{\bar{G}}(\bar{P})$ hence, $\left|\bar{G}: C_{\bar{G}}(\bar{P})\right|=1$ or 3 . Considering that, by the N/C-theorem, $\bar{G} / C_{\bar{G}}(\bar{P})$ is isomorphic to a subgroup of $\operatorname{Aut}(\bar{P})$, and that $\operatorname{Aut}(\bar{P})$ has order 8 , we then have that $\left|\bar{G}: C_{\bar{G}}(\bar{P})\right|=1$. This means that $\bar{P}$ is central in $\bar{G}$. Hence, $\bar{G}$ is the direct product of $\bar{P}$ by a normal subgroup $\bar{O}$ of order 3 which is the quotient by $\Omega$ of a normal subgroup $O$ of $G$ of order $2 n+1$.

Theorem 3.4. There is no 3-pyramidal $\operatorname{STS}(v)$ in each of the following cases:
(i) $v \equiv 1(\bmod 24)$;
(ii) $v \equiv 13(\bmod 24)$;
(iii) $v \equiv 21(\bmod 24)$;
(iv) $v \equiv 27(\bmod 48)$; 
(v) $v \equiv 43(\bmod 48)$.

Proof. Cases (i)-(ii). Suppose that there exists a $\operatorname{STS}(v)$ with $v=24 n+1$ or $v=24 n+13$ which is 3-pyramidal under a group $G$. Therefore $G$ is a group of order $24 n-2$ or $24 n+10$ with exactly three subgroups of order 2 . Now note that these subgroups are precisely the 2-Sylow subgroups of $G$ since the order of $G$ is divisible by 2 but not by 4 . Hence $n_{2}(G)$, the number of 2-Sylow subgroups of $G$, is 3 . By the third Sylow theorem $n_{2}(G)$ should be also a divisor of $\frac{|G|}{2}$. We conclude that 3 should be a divisor of $|G|$ which is clearly false.

Case (iii). Now assume that there exists a 3-pyramidal STS $(24 n+21)$. Then there exists a $\left(G,\left\{2^{3}, 3^{e}\right\}, 3,1\right)$-DF $\mathcal{F}$ for a suitable group $G$ of order $24 n+18$ with exactly 3 involutions and a suitable $e \geq 0$.

By Lemma 3.1 there is a subgroup $S$ of $G$ of index 2. Note that an element of $G$ has odd or even order according to whether it is in $S$ or not, respectively. Thus, in particular, the three involutions of $G$ are all contained in $G \backslash S$ while every subgroup of $G$ of order 3 is contained in $S$. Thus the subset of $G \backslash S$ which is covered by $\Delta \mathcal{F}$ has size $|G \backslash S|-3=$ $12 n+6$.

If $B$ is any block of $\mathcal{F}$ having some differences in $G \backslash S$, then it necessarily has two points lying in distinct cosets of $S$ in $G$. Thus, up to translations, we have $B=\{0, s, t\}$ with $s \in S$ and $t \in G \backslash S$. It follows that $\Delta B \cap(G \backslash S)=\{ \pm t, \pm(s-t)\}$, hence $\Delta B$ has exactly four elements in $G \backslash S$.

From the above two paragraphs we conclude that $12 n+6$ should be divisible by 4 which is clearly absurd.

Cases (iv)-(v). Assume that there exists a 3-pyramidal $\operatorname{STS}(v)$ with $v \equiv 27$ or $43(\bmod$ 48). Thus there exists a $\left(G,\left\{2^{3}, 3^{e}\right\}, 3,1\right)$-DF $\mathcal{F}$ for a suitable group $G$ of order $48 n+24$ or $48 n+40$ with exactly three involutions and where $e>0$ in case (iv) or $e=0$ in case (v). Note that in both cases we have $|G| \equiv 8(\bmod 16)$ so that, by Lemma $3.3, G$ has a subgroup $S$ of index 2 containing exactly one involution. Thus the subset of $G \backslash S$ which is covered by $\Delta \mathcal{F}$ has size $|G \backslash S|-2=24 n+10$ or $24 n+18$. Then, reasoning as in case (iii), $24 n+10$ or $24 n+18$ should be divisible by 4 which is absurd.

\section{The "only if part"}

For proving the "only if part" of our main theorem, we have to give a direct construction for a 3-pyramidal $\operatorname{STS}(v)$ whenever $v$ is admissible and not forbidden by Theorem 3.4, hence for any $v \equiv 7,9,15(\bmod 24)$ and for any $v \equiv 3,19(\bmod 48)$. The most laboured constructions are those for the last two cases where we will use extended Skolem sequences and extended Langford sequences.

Given a pair $(k, n)$ of positive integers with $1 \leq k \leq 2 n+1$, a $k$-extended Skolem sequence of order $n$ can be viewed as a sequence $\left(s_{1}, \ldots, s_{n}\right)$ of $n$ integers such that

$$
\bigcup_{i=1}^{n}\left\{s_{i}, s_{i}+i\right\}=\{1,2, \ldots, 2 n+1\} \backslash\{k\} \text {. }
$$

The existence question for extended Skolem sequences was completely settled by C. Baker in [1].

Theorem 4.1 ([1]). There exists a k-extended Skolem sequence of order $n$ if and only if either $k$ is odd and $n \equiv 0,1(\bmod 4)$ or $k$ is even and $n \equiv 2,3(\bmod 4)$. 
A $k$-extended Langford sequence of order $n$ and defect $d$ can be viewed as a sequence of $n$ integers $\left(\ell_{1}, \ldots, \ell_{n}\right)$ such that

$$
\bigcup_{i=1}^{n}\left\{\ell_{i}, \ell_{i}+i+d-1\right\}=\{1,2, \ldots, 2 n+1\} \backslash\{k\} .
$$

We need the following partial result about extended Langford sequences by V. Linek and S. Mor.

Theorem 4.2 ([11]). There exists a k-extended Langford sequence of order $n$ and defect $d$ for any triple $(k, n, d)$ with $n \geq 2 d, n \equiv 2(\bmod 4)$ and $k$ even.

For general background concerning Skolem sequences, their variants, and their applications, we refer to [20]. We also refer to the recent survey [10] which, however, fails to mention some of our work; for instance, extended Skolem sequences have been crucial in our mentioned work on 1-rotational STSs [2, 4] and also in two papers [6, 22] dealing, more generally, with 1-rotational $k$-cycle systems.

In the next lemma we combine Skolem sequences and Langford sequences to get the last ingredient that we need for proving the "only if part" of our main result. This lemma will be used, specifically, in the construction of a 3 -pyramidal $\operatorname{STS}(v)$ with $v \equiv 3(\bmod$ 96).

Lemma 4.3. There exists a $\left(\mathbb{Z}_{12 n},\{3,4\}, 3,1\right)$-DF for any even $n \geq 2$.

Proof. We have to construct a set $\mathcal{F}_{n}$ of $2 n-1$ triples with elements in $\mathbb{Z}_{12 n}$ whose differences cover $\mathbb{Z}_{12 n} \backslash\{0,3 n, 4 n, 6 n, 8 n, 9 n\}$ exactly once. For the small cases $n \in$ $\{2,4,6,8,12,14,20\}$ one can check that we can take $\mathcal{F}_{n}=\left\{B_{1}, \ldots, B_{2 n-1}\right\}$ with $B_{i}=$ $\left\{0, i, b_{i}\right\}$ and the $b_{i} \mathrm{~s}$ as in the following table:

\begin{tabular}{r|c}
$n$ & $\left(b_{1}, b_{2}, \ldots, b_{2 n-1}\right)$ \\
\hline \hline 2 & $(5,9,13)$ \\
\hline 4 & $(40,37,34,30,38,29,28)$ \\
\hline 6 & $(17,20,22,25,28,33,36,34,39,47,46,52,54,45,53)$ \\
\hline 8 & $(66,63,37,64,38,62,39,58,40,59,41,67,42,68,43,69,44,70,45,71,46,57,47)$ \\
\hline 12 & $(76,74,43,70,44,77,45,73,46,68,47,69,48,78,49,79,50,80,51,81,52,82,53$, \\
& $83,54,67,55)$ \\
\hline 20 & $(110,103,61,108,62,102,63,100,64,105,65,106,66,107,67,98,68,99,69,111$, \\
& $70,112,71,113,72,114,73,115,74,116,75,117,76,118,77,119,78,97,79)$
\end{tabular}

For all the other values of $n$, set $n=2 m$ and $\epsilon=r+(-1)^{r}$ where $r$ is the remainder of the Euclidean division of $m$ by 4 .

By applying Theorem 4.1 one can see that there exists a $(2 m+1)$-extended Skolem sequence $\left(s_{1}, \ldots, s_{m+\epsilon}\right)$ of order $m+\epsilon$. Also, by applying Theorem 4.2 one can see that there exists a $(2 m-2 \epsilon)$-extended Langford sequence $\left(\ell_{1}, \ldots, \ell_{3 m-\epsilon-1}\right)$ of order $3 m-\epsilon-1$ and defect $m+\epsilon+1$. Then one can see that the desired difference family is the one whose blocks are the following:

$$
\begin{aligned}
& \left\{0,-i, s_{i}+4 m-1\right\} \\
& \left\{0,-(m+\epsilon+i), \ell_{i}+6 m+2 \epsilon\right\}
\end{aligned}
$$

with $1 \leq i \leq m+\epsilon$;

with $1 \leq i \leq 3 m-\epsilon-1$. 
Theorem 4.4. There exists a 3-pyramidal $\operatorname{STS}(v)$ for any admissible value of $v$ not forbidden by Theorem 3.4 .

Proof. Considering that the admissible values of $v$ are those congruent to 1 or $3(\bmod 6)$, we have to prove that there exists a 3-pyramidal $\operatorname{STS}(v)$ in the following five cases:

(i) $v \equiv 7(\bmod 24)$;

(ii) $v \equiv 15(\bmod 24)$;

(iii) $v \equiv 9(\bmod 24)$;

(iv) $v \equiv 3(\bmod 48)$;

(v) $v \equiv 19(\bmod 48)$.

Cases (i)-(ii). Assume that $v \equiv 7,15(\bmod 24)$. Let $\mathcal{F}$ be an $(H,\{h\}, 3,1)$-DF with $H=\mathbb{Z}_{6 n+1}$ and $h=1$ if $v=24 n+7$, or $H=\mathbb{Z}_{3} \times \mathbb{Z}_{2 n+1}$ and $h=3$ if $v=24 n+15$. In the former case the existence of $\mathcal{F}$ is guaranteed by Peltesohn's result (see Remark 2.2) while in the latter it is enough to take $\mathcal{F}=\{\{(0,0),(1, i),(1,-i)\} \mid 1 \leq i \leq n\}$.

The group $G:=\mathbb{Z}_{2} \times \mathbb{Z}_{2} \times H$ has order $v-3$ and exactly 3 involutions. Thus, by Theorem 2.1, it is enough to exhibit a $\left(G,\left\{2^{3}, 3^{e}\right\}, 3,1\right)$-DF for some suitable $e$. Such a $\mathrm{DF}$ is, for instance, the one whose blocks are:

$$
\begin{array}{lr}
\{(0,0)\} \times B & \text { with } B \in \mathcal{F} ; \\
\{(0,1,0),(1,0, h),(1,1,-h)\} & \text { with } h \in \bar{H}
\end{array}
$$

where $\bar{H}$ is a subset of $H$ such that $\{\{h,-h\} \mid h \in \bar{H}\}$ is the patterned starter of $H$, i.e., the set of all symmetric 2-subsets of $H$ (see [8]).

Case (iii). Assume that $v \equiv 9(\bmod 24)$, say $v=24 n+9$. The group $G:=\mathbb{D}_{6} \times \mathbb{Z}_{4 n+1}$ has order $v-3$ and clearly has exactly three involutions. Thus, by Theorem 2.1 , it is enough to exhibit a $\left(G,\left\{2^{3}, 3^{1}\right\}, 3,1\right)$-DF (of course here the DF will "use" multiplication on the first component and addition on the second). For this, we have to distinguish three cases according to whether $n$ is odd or congruent to 0 or $2(\bmod 4)$. The three cases are very similar; the blocks of the desired difference family can be taken as indicated in the table below.

\begin{tabular}{l|c|c|c}
\multicolumn{1}{c|}{ Blocks } & $n$ odd & $n \equiv 0(\bmod 4)$ & $n \equiv 2(\bmod 4)$ \\
\hline \hline$\{(1,0),(x, n),(x, 2 n)\}$ & Yes & Yes & Yes \\
\hline$\left\{(y, 0),\left(1,-\frac{n+1}{2}\right),\left(1, \frac{3 n+1}{2}\right)\right\}$ & Yes & No & No \\
\hline$\left\{(1,0),\left(x,-\frac{n}{2}\right),\left(x, \frac{3 n}{2}\right)\right\}$ & No & Yes & Yes \\
\hline$\{(y, 0),(1, i),(1,2 n+1-i)\}$ & $\begin{array}{c}1 \leq i \leq n \\
i \neq \frac{n+1}{2}\end{array}$ & $1 \leq i \leq n$ & $1 \leq i \leq n$ \\
\hline$\left\{(y, 0),(x, i),\left(x^{2},-i\right)\right\}$ & $1 \leq i \leq n$ & $\begin{array}{c}1 \leq i \leq n, \\
i \neq \frac{n}{4}\end{array}$ & $\begin{array}{c}1 \leq i \leq n, \\
\text { and } i=\frac{7 n+2}{4}\end{array}$ \\
\hline$\left\{(y, 0),(x,-i),\left(x^{2}, i\right)\right\}$ & $n+1 \leq i \leq 2 n$ & $\begin{array}{c}n+1 \leq i \leq 2 n \\
\text { and } i=\frac{n}{4}\end{array}$ & $\begin{array}{c}n+1 \leq i \leq 2 n \\
\text { and } i \neq \frac{7 n+2}{4}\end{array}$ \\
\hline$\{(1,0),(x, i),(x, 2 n-i)\}$ & $1 \leq i \leq n-1$ & $\begin{array}{c}1 \leq i \leq n-1 \\
\text { and } i \neq \frac{n}{2}\end{array}$ & $\begin{array}{c}1 \leq i \leq n-1 \\
\text { and } i \neq \frac{n}{2}\end{array}$
\end{tabular}

We note that the subgroup of order 3 which is not covered by the differences of the above families is, in any subcase, $\left\{(1,0),(x, 0),\left(x^{2}, 0\right)\right\}$. 
Case (iv). Assume that $v \equiv 3(\bmod 48)$, say $v=48 n+3$. The group $G=\mathbb{Z}_{4} \times \mathbb{Z}_{12 n}$ has order $v-3$ and it has exactly three involutions. Then, by Theorem 2.1 , it is enough to exhibit a $\left(G,\left\{2^{3}, 3\right\}, 3,1\right)$-DF.

Subcase (iv.1): $n$ is odd. Take a $(2 n+1)$-extended Skolem sequence $\left(s_{1}, \ldots, s_{2 n-1}\right)$ of order $2 n-1$ (which exists by Theorem 4.1). Set $n=2 t+1$ and check that the blocks of a $\left(G,\left\{2^{3}, 3\right\}, 3,1\right)$-DF are the following:

$$
\begin{array}{ll}
\{(0,0),(1,0),(3,6 t+3)\} ; & \\
\{(0,0),(1,3 t+2),(1,-9 t-5)\} ; & \\
\{(0,0),(1, i),(3,12 t+7-i)\} & \text { with } 1 \leq i \leq 6 t+3 \text { and } i \neq 3 t+2 ; \\
\{(0,0),(1,6 t+3+i),(3,6 t+3-i)\} & \text { with } 1 \leq i \leq 6 t+2 ; \\
\left\{(0,0),(0, i),\left(0,-s_{i}-4 t-1\right)\right\} & \text { with } 1 \leq i \leq 4 t+1 .
\end{array}
$$

Subcase (iv.2): $n$ is even. Take a $\left(\mathbb{Z}_{12 n},\{3,4\}, 3,1\right)$-DF $\mathcal{F}$ using Lemma 4.3. One can see that a $\left(G,\left\{2^{3}, 3\right\}, 3,1\right)$-DF is the one whose blocks are the following.

$$
\begin{array}{ll}
\{(0,0),(1,0),(1,9 n)\} ; & \\
\{0\} \times B & \text { with } B \in \mathcal{F} ; \\
\{(0,0),(1, i),(3,6 n+1-i)\} & \text { with } 1 \leq i \leq 3 n ; \\
\{(0,0),(1, i),(3,6 n-i)\} & \text { with } 3 n+1 \leq i \leq 6 n-1 .
\end{array}
$$

Case (v). Assume that $v \equiv 19(\bmod 48)$, say $v=48 n+19$. The group $G=\mathbb{Z}_{4} \times$ $\mathbb{Z}_{12 n+4}$ has order $v-3$ and it has exactly three involutions. Then, by Theorem 2.1 , it is enough to exhibit a $\left(G,\left\{2^{3}\right\}, 3,1\right)$-DF. Let $\left(s_{1}, s_{2}, \ldots, s_{2 n}\right)$ be any $(n+1)$-extended Skolem sequence of order $2 n$ (which exists by Theorem 4.1). Then the required difference family is the one whose blocks are the following:

$$
\begin{array}{ll}
\{(0,0),(1,0),(1,3 n+1)\} ; & \\
\left\{(0,0),(0, i),\left(0,-s_{i}-2 n\right)\right\} & \text { with } 1 \leq i \leq 2 n ; \\
\{(0,0),(1, i),(3,6 n+2-i)\} & \text { with } 1 \leq i \leq 3 n ; \\
\{(0,0),(1,6 n+3-i),(3, i)\} & \text { with } 1 \leq i \leq 3 n+1 .
\end{array}
$$

\section{Conclusion}

Theorem 3.4 and Theorem 4.4 are the "if part" and the "only if part" of the main result Theorem 1.2 which therefore is now completely proved.

The existence of a 3-pyramidal STS $(v)$ can be summarized in the following table where, in the third column, we put a group acting 3-pyramidally on a $\operatorname{STS}(v)$. 


\begin{tabular}{l|c|c}
$v$ & Existence & Group \\
\hline \hline $24 n+1$ & No & - \\
\hline $24 n+3$ & Yes $\Longleftrightarrow n$ is even & $\mathbb{Z}_{4} \times \mathbb{Z}_{6 n}$ \\
\hline $24 n+7$ & Yes & $\mathbb{Z}_{2}^{2} \times \mathbb{Z}_{6 n+1}$ \\
\hline $24 n+9$ & Yes & $\mathbb{D}_{6} \times \mathbb{Z}_{4 n+1}$ \\
\hline $24 n+13$ & No & - \\
\hline $24 n+15$ & Yes & $\mathbb{Z}_{2}^{2} \times \mathbb{Z}_{3} \times \mathbb{Z}_{2 n+1}$ \\
\hline $24 n+19$ & Yes $\Longleftrightarrow n$ is even & $\mathbb{Z}_{4} \times \mathbb{Z}_{6 n+4}$ \\
\hline $24 n+21$ & No & -
\end{tabular}

Note that an abelian group of order $24 n+6$ has only one involution so that there is no $\operatorname{STS}(24 n+9)$ which is 3-pyramidal under an abelian group. Thus we see, from the above table, that there exists a $\operatorname{STS}(v)$ which is 3-pyramidal under an abelian group if and only if $v \equiv 7,15(\bmod 24)$ or $v \equiv 3,19(\bmod 48)$.

\section{References}

[1] C. A. Baker, Extended Skolem sequences, J. Combin. Des. 3 (1995), 363-379, doi:10.1002/ jcd.3180030507.

[2] S. Bonvicini, M. Buratti, G. Rinaldi and T. Traetta, Some progress on the existence of 1-rotational Steiner triple systems, Des. Codes Cryptogr. 62 (2012), 63-78, doi:10.1007/ s10623-011-9491-3.

[3] M. Buratti, Recursive constructions for difference matrices and relative difference families, J. Combin. Des. 6 (1998), 165-182, doi:10.1002/(sici)1520-6610(1998)6:3〈165::aid-jcd1〉3.0. co;2-d.

[4] M. Buratti, 1-rotational Steiner triple systems over arbitrary groups, J. Combin. Des. 9 (2001), 215-226, doi:10.1002/jcd.1008.abs.

[5] M. Buratti, Constructions for point-regular linear spaces, J. Statist. Plann. Inference 94 (2001), 139-146, doi:10.1016/s0378-3758(00)00247-0.

[6] M. Buratti, Existence of 1-rotational $k$-cycle systems of the complete graph, Graphs Combin. 20 (2004), 41-46, doi:10.1007/s00373-003-0547-7.

[7] C. J. Colbourn and A. Rosa, Triple Systems, Oxford Mathematical Monographs, Clarendon Press, Oxford, 1999.

[8] J. H. Dinitz, Starters, in: C. J. Colbourn and J. H. Dinitz (eds.), Handbook of Combinatorial Designs, Chapman \& Hall/CRC, Boca Raton, Florida, Discrete Mathematics and its Applications, chapter 55, pp. 622-628, 2nd edition, 2007, doi:10.1201/9781420049954.

[9] J. Doyen, A note on reverse Steiner triple systems, Discrete Math. 1 (1972), 315-319, doi: $10.1016 / 0012-365 x(72) 90038-6$.

[10] N. Francetić and E. Mendelsohn, A survey of Skolem-type sequences and Rosa's use of them, Math. Slovaca 59 (2009), 39-76, doi:10.2478/s12175-008-0110-3.

[11] V. Linek and S. Mor, On partitions of $\{1, \ldots, 2 m+1\} \backslash\{k\}$ into differences $d, \ldots, d+$ $m-1$ : Extended Langford sequences of large defect, J. Combin. Des. 12 (2004), 421-442, doi:10.1002/jcd.20001.

[12] G. J. Lovegrove, The automorphism groups of Steiner triple systems obtained by the Bose construction, J. Algebraic Combin. 18 (2003), 159-170, doi:10.1023/b:jaco.0000011935.37751. c5. 
[13] E. Mendelsohn, On the groups of automorphisms of Steiner triple and quadruple systems, $J$. Combin. Theory Ser. A 25 (1978), 97-104, doi:10.1016/0097-3165(78)90072-9.

[14] M. Mishima, The spectrum of 1-rotational Steiner triple systems over a dicyclic group, Discrete Math. 308 (2008), 2617-2619, doi:10.1016/j.disc.2007.06.001.

[15] R. Peltesohn, Eine Lösung der beiden Heffterschen Differenzenprobleme, Compositio Math. 6 (1939), 251-257.

[16] K. T. Phelps and A. Rosa, Steiner triple systems with rotational automorphisms, Discrete Math. 33 (1981), 57-66, doi:10.1016/0012-365x(81)90258-2.

[17] A. Pott, A survey on relative difference sets, in: Groups, Difference Sets, and the Monster, De Gruyter, Berlin, volume 4 of Ohio State Univ. Math. Res. Inst. Publ., pp. 195-232, 1996, doi:10.1515/9783110893106.195.

[18] A. Rosa, On reverse Steiner triple systems, Discrete Math. 2 (1972), 61-71, doi:10.1016/ 0012-365x(72)90061-1.

[19] J. S. Rose, A Course on Group Theory, Cambridge University Press, Cambridge, 1978.

[20] N. Shalaby, Skolem and Langford sequences, in: C. J. Colbourn and J. H. Dinitz (eds.), Handbook of Combinatorial Designs, Chapman \& Hall/CRC, Boca Raton, Florida, Discrete Mathematics and its Applications, chapter 53, pp. 612-616, 2nd edition, 2007, doi: $10.1201 / 9781420049954$.

[21] L. Teirlinck, The existence of reverse Steiner triple systems, Discrete Math. 6 (1973), 301-302, doi:10.1016/0012-365x(73)90102-7.

[22] S.-L. Wu and M. Buratti, A complete solution to the existence problem for 1-rotational $k$-cycle systems of $K_{v}$, J. Combin. Des. 17 (2009), 283-293, doi:10.1002/jcd.20217. 